\title{
SOME LAGRANGIAN INVARIANTS OF SYMPLECTIC MANIFOLDS
}

\author{
MICHEL NGUIFFO BOYOM \\ UMR CNRS 5149, Département de Mathématiques, Université Montpellier II \\ Montpellier, France \\ E-mail: boyom@math.univ-montp2.fr
}

\begin{abstract}
The KV-homology theory is a new framework which yields interesting properties of lagrangian foliations. This short note is devoted to relationships between the KV-homology and the KV-cohomology of a lagrangian foliation. Let us denote by $\mathcal{A}_{F}$ (resp. $V^{F}$ ) the $\mathrm{KV}$-algebra (resp. the space of basic functions) of a lagrangian foliation $F$. We show that there exists a pairing of cohomology and homology to $V^{F}$. That is to say, there is a bilinear map $H^{q}\left(\mathcal{A}_{F}, V^{F}\right) \times$ $H_{q}\left(\mathcal{A}_{F}, V^{F}\right) \rightarrow V^{F}$, which is invariant under $F$-preserving symplectic diffeomorphisms.
\end{abstract}

Introduction. A locally flat structure on a manifold $M$ is a pair $(M, D)$ where $D$ is a torsion free linear connection whose curvature tensor vanishes identically, [NB2,4], $[\mathrm{KH}]$, [WR1]. A real valued function $f$ defined on $M$ is called an affine function if its D-hessian vanishes identically. This means that the covariant derivative $D(d f)$ of $d f$ vanishes identically. Every locally flat structure $(M, D)$ gives rise to an atlas $A t=\left(U_{\alpha}, \phi_{\alpha}\right)$ whose local coordinate functions are affine functions. The pair $(M, A t)$ is called an affinely flat structure on $M$. The space $\mathcal{X}(M)$ of smooth vector fields on a locally flat manifold is a Koszul-Vinberg algebra (shortly $\mathrm{KV}$-algebra). We denote it by $\mathcal{A}$. This note is devoted to the real valued homology of $\mathcal{A}$, [NB6]. This homology and the real valued KV-cohomology are paired to the space of real valued functions. The case of $\mathrm{KV}$-algebras of lagrangian foliations is studied. These foliations played important roles in many mathematical problems and in mathematical physics as well, [GLS], [HH], [KB], [MJ], [SJ], [SW], [WR1,2]. We also overview some techniques such as spectral sequences of KV-cohomology which are clearly affine invariants. This material can be used to investigate new invariants and new properties of affinely flat manifolds and of lagrangian foliations on symplectic manifolds. The details will appear in [NB6].

2000 Mathematics Subject Classification: Primary 55N35, 53B05, 55T10; Secondary 17B40, 17B55

Key words and phrases: KV-algebra, KV-homology, pairing, spectral sequence.

The paper is in final form and no version of it will be published elsewhere. 


\section{Algebra}

1.1. Main definitions. All vector spaces have the same commutative ground field $\mathbb{F}$ whose characteristic is zero. When there is no risk of confusion the multiplication map of an algebra $\mathcal{A}$ is denoted by $a b \forall a, b \in \mathcal{A}$. A left action (resp. right action) of $\mathcal{A}$ on an $\mathcal{A}$-module $V$ is denoted by $a v$ (respectively by $v a) \forall a \in \mathcal{A}, \forall v \in V$.

Definition 1 . An algebra $\mathcal{A}$ is called a $K V$-algebra if its multiplication map satisfies the identity $a(b c)-(a b) c=b(a c)-(b a) c$.

Koszul-Vinberg algebras are called Vinberg algebras in [NA]. They are also called "Pre-Lie algebras" [CL], [MS] or "left symmetric algebras" [NB], [KH]. Mutatis mutandis, an algebra whose associator is right symmetric is called a "right symmetric algebra", [DA]. There is no relevant difference between left symmetric algebras and right symmetric algebras. The pioneering work on the cohomology theory of Koszul-Vinberg algebra is due to Albert Nijenhuis, [NA]. For a complete account on the KV-cohomology theory the reader is referred to [NB3]. Another presentation of the pioneering result of [NA] has been given by Chapoton and Livernet [CL] (see also [DA]).

Definition 2 ([NB3]). A vector space $V$ endowed with a two-sided action of $\mathcal{A}$ is called a two-sided module over the $\mathrm{KV}$-algebra $\mathcal{A}$ if the following identities hold: (i) $a(b v)-(a b) v=$ $b(a v)-(b a) v$, (ii) $a(v b)-(a v) b=v(a b)-(v a) b, \forall a, b \in \mathcal{A}, \forall v \in V$.

Let $V$ and $W$ be two-sided modules over the $\mathrm{KV}$-algebra $\mathcal{A}$. The tensor product $V \otimes W$ is a two-sided module over $\mathcal{A}$ under the following actions

$$
\begin{gathered}
a(v \otimes w)=a v \otimes w+v \otimes a w, \\
(v \otimes w) a=v \otimes w a .
\end{gathered}
$$

The space $L(V, W)$ of $W$-valued linear functions on $V$ is a two-sided module over $\mathcal{A}$ under the following actions of $\mathcal{A}$

$$
\begin{gathered}
(a f)(v)=a(f(v))-f(a v), \\
(f a)(v)=(f(v)) a .
\end{gathered}
$$

Given a two-sided module $V$ over a $\mathrm{KV}$-algebra $\mathcal{A}$ the subspace $J(V)$ consists in elements $v \in V$ satisfying the identity $a(b v)=(a b) v, \forall a, b \in \mathcal{A}$.

ExAMPLE 1. Associative algebras are KV-algebras.

ExAmple 2. The space $\mathcal{X}\left(S^{1}\right)$ of smooth vector fields on the circle is a $\mathrm{KV}$-algebra whose multiplication is defined by

$$
f(\exp (i \pi t)) \frac{d}{d t} \cdot g(\exp (i \pi t)) \frac{d}{d t}=f(\exp (i \pi t)) \frac{d g(\exp (i \pi t))}{d t} \frac{d}{d t} .
$$

The associator to this multiplication is:

$$
\begin{aligned}
& \left(f(\exp (i \pi t)) \frac{d}{d t}, g(\exp (i \pi t)) \frac{d}{d t}, h(\exp (i \pi t)) \frac{d}{d t}\right) \\
& =f(\exp (i \pi t)) g(\exp (i \pi t)) \frac{d^{2} h(\exp (i \pi t))}{d t^{2}} \frac{d}{d t} .
\end{aligned}
$$


ExAmPLE 3. Many interesting KV-algebras come from differential geometry. For instance let $(M, D)$ be a pair where $M$ is a smooth manifold and $D$ is a torsion free linear connection whose curvature tensor vanishes identically. The space $\mathcal{X}(M)$ of smooth vector fields on $M$ is a $\mathrm{KV}$-algebra under the multiplication defined by $X Y=D_{X} Y$. This $\mathrm{KV}$-algebra is denoted by $\mathcal{A}$. It is called the $\mathrm{KV}$-algebra of $(M, D)$.

Another situation we shall deal with is the following.

ExAmple 4. Let $(M, \omega)$ be a symplectic manifold. Let $X, Y \in \mathcal{X}(M)$. Then we define the multiplication $X \diamond Y \in \mathcal{X}(M)$ by the formula $i_{X \diamond Y} \omega=L_{X} i_{Y} \omega$ where $i_{Y}$ is the inner product by $Y$ and $L_{X}$ is the Lie derivation in the direction of $X$. Thus $(\mathcal{X}(M), \diamond)$ is a real algebra with a nice geometric property, namely: The multiplication $X \diamond Y$ preserves every lagrangian foliation of $(M, \omega)$; moreover with this multiplication $\diamond$ the space of tangent vector fields of such a foliation is a $\mathrm{KV}$-algebra.

Examples of modules:

(i) A KV-algebra is a two-sided module over itself.

(ii) Considering Example 3, the vector space $T(M)$ of tensors fields on $M$ is a two-sided module over $\mathcal{A}$.

1.2. $K V$-Homology. The aim of this subsection is to recall the new homology complex of $\mathrm{KV}$-algebras with coefficients in their two-sided modules, [NB6]. In general the homology complex that we are going to define is not the algebraic dual of the cohomology complex which has been studied in [NB3].

Let $V$ be a two-sided module over a KV-algebra $\mathcal{A}$. Let $q$ be a positive integer. Then $\mathcal{A}^{\otimes q}$ stands for the $q^{t h}$ tensor power of the vector space $\mathcal{A}$. Let $\xi=a_{1} \otimes . . \otimes a_{q} \in \mathcal{A}^{\otimes q}$. Given $1 \leq i, j \leq q$ we set

$$
\partial_{i} \xi=a_{1} \otimes . . \otimes \hat{a}_{i} . . \otimes a_{q}=a_{1} \otimes . . \otimes a_{i-1} \otimes a_{i+1} \otimes . . \otimes a_{q} .
$$

Let $J(V)=v \in V / a(b v)=(a b) v, \forall a, b \in \mathcal{A}$

Now we consider the $Z$-graded vector space

$$
C(\mathcal{A}, V)=\sum_{q} C_{q}(\mathcal{A}, V)
$$

with $C_{q}(\mathcal{A}, V)=0$ if $q$ is negative, $C_{0}(\mathcal{A}, V)=J(V)$ and $C_{q}(\mathcal{A}, V)=\mathcal{A}^{\otimes q} \otimes V$ whenever $q$ is positive. We define the linear maps

$$
d_{q}: C_{q}(\mathcal{A}, V) \rightarrow C_{q-1}(\mathcal{A}, V)
$$

as it follows: $d_{q}=0$ if $\leq 1$. If $q \geq 2$, take $\eta=\xi \otimes v \in C_{q}(\mathcal{A}, V)$ with $\xi=a_{1} \otimes . . \otimes a_{q}$. Define

$$
d_{q} \eta=\sum_{i \leq q-1}(-1)^{i}\left[\partial_{i} \xi \otimes v a_{i}-a_{i}\left(\partial_{i} \xi \otimes v\right)+\left(\partial_{i q}^{2} \xi\right) \otimes a_{i} \otimes v a_{q}\right]
$$

LEMMA 1. The operators $d_{q}$ satisfy the identity $d_{q-1} d_{q}=0$.

The proof of the main lemma above consists of straightforward careful calculations. This lemma yields the following chain complex

$$
\rightarrow C_{q+1}(\mathcal{A}, V) \rightarrow C_{q}(\mathcal{A}, V) \rightarrow C_{q-1}(\mathcal{A}, V) \rightarrow
$$

whose $q^{t h}$ homology space is denoted by $H_{q}(\mathcal{A}, V)$. 
ExAmple 1 . Let $\mathcal{A}$ be the algebra structure on $\mathbb{F}^{2}$ defined by

$$
(x, y) \cdot\left(x^{\prime}, y^{\prime}\right)=\left(x x^{\prime}, o\right) .
$$

It is an associative algebra. Regarding $\mathbb{F}$ as trivial module over $\mathcal{A}$ we identify $C_{2}(\mathcal{A}, \mathbb{F})$ with the space $\mathcal{M}(2, \mathbb{F})$ of matrices $\left[a_{i j}\right], i, j:=1,2$. The space of 2 -cycles is defined by $a_{11}=0$ and the subspace of 2-boundaries is defined by $a_{11}=a_{21}=0$. So $H_{2}(\mathcal{A}, \mathbb{F})$ is two dimensional.

EXAmple 2. Consider the subspace $\mathcal{A} \subset \mathcal{M}(3, \mathbb{F})$ consisting of the nilpotent upper triangular matrices. Identify $\mathcal{A}$ with $\mathbb{F}^{3}$ endowed with the multiplication $(x, y, z)\left(x^{\prime}, y^{\prime}, z^{\prime}\right)=$ $\left(0,0, x y^{\prime}\right)$. This is an associative algebra. We identify $C_{2}(\mathcal{A}, \mathbb{F})$ with the space $\mathcal{M}(3, \mathbb{F})$ of square matrices $\left[a_{i j}\right], i, j=1,2,3$. The vector space of 2 -cycles is defined by $a_{12}=0$ while the subspace of 2-boundaries is defined by the system $a_{11}=a_{12}=a_{21}=a_{22}=0$, $a_{23}=a_{32}$. Thereby the vector space $H_{2}(\mathcal{A}, \mathbb{F})$ is the subspace of $\mathcal{M}(3, \mathbb{F})$ defined by the system $a_{12}=a_{31}=a_{33}=0, a_{23}+a_{32}=0$.

1.3. $K V$-cohomology. In this subsection we recall the definition of the cohomology complex of $\mathrm{KV}$-algebras with coefficients in their two-sided modules, see [NB3], [NB4]. Let $V$ be a two-sided module over a $\mathrm{KV}$-algebra $\mathcal{A}$. The $Z$-graded cochain complex

$$
\rightarrow C^{q-1}(\mathcal{A}, V) \rightarrow C^{q}(\mathcal{A}, V) \rightarrow C^{q+1}(\mathcal{A}, V) \rightarrow
$$

is defined by $C^{q}(\mathcal{A}, V)=0$ if $q$ is negative, $C^{0}(\mathcal{A}, V)=J(V)$ and $C^{q}(\mathcal{A}, V)=\operatorname{Hom}\left(\mathcal{A}^{\otimes q}, V\right)$ if $q$ is positive. The coboundary operators

$$
d_{q}: C^{q}(\mathcal{A}, V) \rightarrow C^{q+1}(\mathcal{A}, V)
$$

are defined as follows. First, $\left(d_{0} v\right)(a)=-a v+v a$. If $q$ is positive, let $f \in C^{q}(\mathcal{A}, V)$ and $\xi=a_{1} \otimes . . \otimes a_{q+1} \in \mathcal{A}^{\otimes q+1}$, one sets

$$
\left(d_{q} f\right)(\xi)=\sum_{i \leq q}(-1)^{i}\left[\left(a_{i} f\right)\left(\partial_{i} \xi\right)+\left(f a_{q+1}\right)\left(\partial_{i q+1}^{2} \xi \otimes a_{i}\right)\right] .
$$

These operators satisfy the identity $d_{q+1} d_{q}=0$. The induced cohomology provides the solution of a conjecture of Gerstenhaber for the theory of deformation of Koszul-Vinberg algebras, see [MG], [NB3]. It also explains why hyperbolic locally flat manifolds always admit nontrivial deformations, [KJL], [NB3].

1.4. Pairing. Let $V$ be a two-sided module over a $\mathrm{KV}$-algebra $\mathcal{A}$. Regarding the base field $\mathbb{F}$ as a trivial module over $\mathcal{A}$, the dual vector space $V^{\star}=\operatorname{Hom}(V, \mathbb{F})$ is a left module over $\mathcal{A}$. Let us consider the canonical pairing of $V^{\star} \times V$ to $\mathbb{F}$, [HS]. It yields a pairing of $C^{q}\left(\mathcal{A}, V^{\star}\right) \times C_{q}(\mathcal{A}, V)$ to $\mathbb{F}$. However, it is easy to check by a direct calculation that the coboundary operator

$$
d_{q}: C^{q}\left(\mathcal{A}, V^{\star}\right) \rightarrow C^{q+1}\left(\mathcal{A}, V^{\star}\right)
$$

is not the transpose of the boundary operator

$$
d_{q+1} ; C_{q+1}(\mathcal{A}, V) \rightarrow C_{q}(\mathcal{A}, V),
$$

because the starting pairing doesn't say anything at the homology level. Nevertheless by restricting to left modules one has 
THEOREM 1. Let $V$ be a left module over a $K V$-algebra $\mathcal{A}$ and let $V^{\star}=\operatorname{Hom}(V, \mathbb{F})$ be its dual vector space. The canonical pairing of $V^{\star} \times V$ to the base field $\mathbb{F}$ induces a pairing of $H^{q}\left(\mathcal{A}, V^{\star}\right) \times H_{q}(\mathcal{A}, V)$ to $\mathbb{F}$.

Idea of proof. Denote by $v^{\star}, v \rightarrow\left\langle v^{\star}, v>\right.$ the canonical pairing of $V^{\star} \times V$ to $\mathbb{F}$. Let $\theta \in C^{q}\left(\mathcal{A}, V^{\star}\right), \xi=a_{1} \otimes . . \otimes a_{i} . . \otimes a_{q}$ and $\eta=\xi \otimes v \in C_{q}\left(\mathcal{A}, V^{\star}\right)$. We extend the pairing $<v^{\star}, v>$ to $C^{q}\left(\mathcal{A}, V^{\star}\right) \times C_{q}\left(\mathcal{A}, V^{\star}\right)$ by setting $<\theta, \eta>=<\theta(\xi), v>$. When $V$ is a left $\mathrm{KV}$-module of $\mathcal{A}$, we have $<d_{q} \theta, \eta>=<\theta, d_{q+1} \eta>, \forall \theta \in C^{q}\left(\mathcal{A}, V^{\star}\right), \forall \eta \in C_{q+1}\left(\mathcal{A}, V^{\star}\right)$, the details will appear elsewhere.

The aim of the next subsection is to overview some geometric applications of this pairing theorem.

EXAMPLE. We consider the algebra of upper nilpotent triangular matrices of $\mathcal{M}(3, \mathbb{F})$. We identify both $C^{2}(\mathcal{A}, \mathbb{F})$ and $C_{2}(\mathcal{A}, \mathbb{F})$ with $\mathcal{M}(3, \mathbb{F})$. Under this identification the vector spaces $H^{2}(\mathcal{A}, \mathbb{F})$ and $H_{2}(\mathcal{A}, \mathbb{F})$ coincide. So the canonical inner product $(A, B)=$ $\operatorname{trace}\left(A B^{t}\right)$ yields the required result ( $B^{t}$ stands for the transpose of the matrix $B$ ).

2. Differential geometry. In this section we deal with smooth manifolds. All of geometric objects are smooth as well. All of our results hold on holomorphic manifolds whenever smooth geometric objects we deal with have holomorphic analogues.

Let $F$ be a foliation on a smooth manifold $M$. Denote by $\mathcal{X}_{F}$ the space of smooth vector fields which are tangent to $F$ everywhere. It is a subalgebra of the Lie algebra $\mathcal{A}(M)$ of smooth vector fields on $M$.

DEFinition 3. An affine foliation in a smooth manifold $M$ is a smooth foliation $F$ such that $\mathcal{X}_{F}$ is endowed with a $\mathrm{KV}$-algebra structure whose commutator Lie algebra is a subalgebra of the Lie algebra $\mathcal{A}(M)$ of smooth vector fields.

Actually leaves of an affine foliation are locally flat manifolds. Given a regular foliation $F$ let $T F \subset T M$ be the tangent bundle of $F$. Then $F$ is an affine foliation if and only if $T F$ is a Koszul-Vinberg algebroid whose anchor map is the inclusion map, [NB4], [NB5], [NBW1], [NBW2]. Here are some interesting examples of affine foliations.

EXAmple 1. Let $X$ be a smooth vector field on $M$. We denote by $\mathcal{A}$ the vector space consisting of the vector fields $f X$ where $f$ is a smooth function on $M$. Then $\mathcal{A}$ is $\mathrm{KV}$-algebra under the multiplication defined by $(f X)(g X)=f(d g(X)) X$. The set of maximal integral curves of $X$ define an affine foliation. (This foliation may be singular). For instance the Reeb vector field of a contact form defines an affine foliation.

ExAmPle 2. Let $(M, \omega)$ be a symplectic manifold. Then every lagrangian foliation $F$ is an affine foliation. The multiplication of $\mathcal{A}=\mathcal{X}_{F}$ is defined by the formula

$$
i_{X Y} \omega=L_{X} i_{Y} \omega \text {. }
$$

Of course the space $\mathcal{X}(M)$ is a left module over $\mathcal{A}$. The left action of $\mathcal{A}$ on $\mathcal{X}(M)$ is defined by the same formula as above, namely $i_{X Y} \omega=L_{X} i_{Y} \omega \forall X \in \mathcal{A}, \forall Y \in \mathcal{X}(M)$. The space of real-valued smooth functions on $M$ is a left $\mathcal{A}$-module as well. 
From now on let $F$ be a fixed lagrangian foliation in a $2 m$-dimensional symplectic manifold $(M, \omega)$. The $\mathrm{KV}$-algebra of $F$ is denoted by $\mathcal{A}_{F}$. Let $\mathcal{G}_{\omega}$ be the group of symplectomorphisms of $(M, \omega)$ and let $G_{m, 2 m}(T M)$ be the $(m, 2 m)$-grassmannian bundle of $T M$. An element $\mathcal{E}$ of $G_{m, 2 m}(T M)$ is a sub-bundle of the tangent bundle $T M$. Thus $G_{m, 2 m}(T M)$ contains the subbundle $L(\omega)$ of lagrangian foliations on $(M, \omega)$. The natural action of $\mathcal{G}_{\omega}$ in $G_{m, 2 m}(T M)$ is an $L(\omega)$-preserving action. Our aim is to point out a pairing which is a $\mathcal{G}_{\omega}$-invariant on each orbit $\mathcal{G}_{\omega}(F)$ of $\mathcal{G}_{\omega}$ in $L(\omega)$. For $F \in L(\omega)$, the space $V^{F}$ of basic functions of $F$ is a trivial left module over the KV-algebra $\mathcal{A}_{F}$. Remark that the vector space $V^{F}$ may consist only of constant functions. Such is the case if $F$ admits a dense leaf. To $\phi \in \mathcal{G}_{\omega}$ we assign its differential $\phi_{\star}$, its codifferential $\phi^{\star}$ and the pair $\rho_{\phi}=\left(\phi_{\star},\left(\phi^{\star}\right)^{-1}\right)$. One has $\left(\phi^{\star}\right)^{-1}\left(V^{F}\right)=V^{\phi_{\star}(F)}$ and $\phi_{\star}\left(\mathcal{A}_{F}\right)=\mathcal{A}_{\phi_{\star}(F)}$. Owing to these observations, it is easy to verify that the pair $\rho_{\phi}$ gives rise to the following isomorphisms:

$$
\rho_{\phi}: H^{q}\left(\mathcal{A}_{F}, V^{F}\right) \rightarrow H^{q}\left(\mathcal{A}_{\phi(F)}, V^{\phi(F)}\right)
$$

and

$$
\rho_{\phi}: H_{q}\left(\mathcal{A}_{F}, V^{F}\right) \rightarrow H_{q}\left(\mathcal{A}_{\phi(F)}, V^{\phi(F)}\right) .
$$

Keeping these notations one has the following statement:

THEOREM 2. The usual multiplication of real valued functions induces a pairing

$$
\mu_{F}: H^{q}\left(\mathcal{A}_{F}, V^{F}\right) \times H_{q}\left(\mathcal{A}_{F}, V^{F}\right) \rightarrow V^{F} .
$$

Moreover, every $\phi \in \mathcal{G}_{\omega}$ induces the commutative diagram $\left(\phi^{\star}\right)^{-1} \mu_{F}=\mu_{\phi_{\star}(F)}\left(\rho_{\phi} \times \rho_{\phi}\right)$.

To wind up considerations about the action of $\mathcal{G}$ on $L(\omega)$, we suppose $M$ to be compact. The measure $d \nu=\omega^{m}$ yields the $\mathcal{G}_{\omega}$-invariant integral

$$
\int_{M} f(x) d \nu(x)
$$

where $f$ is a real valued smooth function on $M$. Now to every pair $(\theta, \eta) \in H^{q}\left(\mathcal{A}_{F}, V^{F}\right) \times$ $H_{q}\left(\mathcal{A}_{F}, V^{F}\right)$, we assign the integral

$$
I_{F}=\int_{M} \mu_{F}(\theta, \eta) d \nu
$$

The theorem above has the following corollary.

THEOREM 3. The integral $I_{F}$ is constant on the orbit $\mathcal{G}_{\omega}(F)$.

The last statement means that $\forall \phi \in \mathcal{G}_{\omega}, \forall(\theta, \eta) \in H^{q}\left(\mathcal{A}_{F}, V^{F}\right) \times H_{q}\left(\mathcal{A}_{F}, V^{F}\right)$ one has

$$
\int_{M} \mu_{F}(\theta, \eta) d \nu=\int_{M} \mu_{\phi_{\star}(F)}(\rho(\theta), \rho(\eta)) d \nu .
$$

2.1. Legendrian invariant. Many considerations we have been concerned with have their equivalent on manifolds with a contact form. In particular legendrian foliations would play a role which is similar to the role played by lagrangian foliations. The KV-algebra $\mathcal{A}_{\alpha}$ of a contact form $\alpha$ is the $C^{\infty}(M, \mathbb{R})$-module generated by the Reeb vector field $R_{\alpha}$. Its multiplication is defined by

$$
\left(f R_{\alpha}\right)\left(g R_{\alpha}\right)=f d g\left(R_{\alpha}\right) R_{\alpha}
$$


Let $\mathcal{G}_{\alpha}$ be the group of $\alpha$-preserving diffeomorphisms of $M$. Set $2 m+1=\operatorname{dimM}$. Let $d \mu$ be the measure defined by the volume form $\alpha \wedge d \alpha^{m}$. This measure is $\mathcal{G}_{\alpha}$-invariant. Let $V$ be the associative algebra of real valued smooth functions on $M$. It is a left module over $\mathcal{A}_{\alpha}$. Thus on the KV-homology level we get a natural pairing

$$
\beta: H^{q}\left(\mathcal{A}_{\alpha}, V\right) \times H_{q}\left(\mathcal{A}_{\alpha}, V\right) \rightarrow V .
$$

Suppose $M$ to be compact, then we get the real valued bilinear form $(\theta, \eta) \in H^{q}\left(\mathcal{A}_{\alpha}, V\right) \times$ $H_{q}\left(\mathcal{A}_{\alpha}, V\right) \rightarrow \int_{M} \beta(\theta, \eta) d \mu$. This integral is invariant by the group $\mathcal{G}_{\alpha}$, it is a contact invariant.

Considerations above might yield new invariants which depend only on the conformal class of the contact form $\alpha$.

3. Bilagrangian geometry. In this section we intend to outline some questions which deserve to be deeply studied. Let $(M, \omega)$ be a symplectic manifold and let $W$ be the space of real valued smooth functions on $M$. To the $\mathrm{KV}$-algebra $\mathcal{A}$ of a lagrangian foliation $F$, we assign the $\mathrm{KV}$-algebra $\mathcal{B}=\mathcal{A} \oplus W$ whose multiplication is defined as follows:

$$
(a, w) \cdot\left(a^{\prime}, w^{\prime}\right)=\left(a a^{\prime}, a w^{\prime}+w w^{\prime}\right) .
$$

In the right side of the formula above, $a w^{\prime}$ stands for the Lie derivative of $w^{\prime}$ in the direction of $a$ and $w w^{\prime}$ is the usual product of two real valued functions. We have mentioned that the space $\mathcal{X}(M)$ of smooth vector fields is a left module over $\mathcal{A}_{F}$. Actually $\mathcal{A}_{F}$ is a left ideal of the $\mathrm{KV}$-algebra $\mathcal{B}$. The relative cochain complex $C\left(\mathcal{B}, \mathcal{A}_{F} ; W\right)$ is defined similarly to the Chevalley-Eilenberg theory. As will be seen below its cohomology space may be regarded as the term $E_{1}$ of a spectral sequence, [HS]. An $\mathcal{A}_{F}$-relative $q$-cochain is a cochain $\theta$ satisfying the identities $e_{j}(a) \theta=0$ and $e_{j}(a) d \theta=0, \forall a \in \mathcal{A}, \forall 0 \leq j \leq q+1$.

3.1. Spectral sequences. According to the notations used in the preceding subsection, we equip the complex $C^{\star}(\mathcal{B}, W)$ with the filtration

$$
F^{j} C(\mathcal{B}, W)=\sum_{q} F^{j} C(\mathcal{B}, W) \cap C^{q}(\mathcal{B}, W)
$$

$\theta \in F^{j} C(\mathcal{B}, W) \cap C^{q}(\mathcal{B}, W)$ iff $e\left(\mathcal{A}^{\otimes q-j+1}\right) \theta=0$. That is, $\theta\left(x_{1}, . ., x_{q}\right)=0$, whenever more than $q-j+1$ arguments belong to $\mathcal{A}$. Thus by setting $C^{q-r, r}(\mathcal{B}, W)=\operatorname{Hom}\left(\mathcal{A}^{\otimes q-r} \otimes\right.$ $\left.W^{r}, W\right)$ we get

$$
F^{j} C(\mathcal{B}, W) \cap C^{q}(\mathcal{B}, W)=\sum_{j \leq r \leq q} C^{q-r, r}(\mathcal{B}, W) .
$$

This filtration satisfies the conditions

$$
F^{j+1} C(\mathcal{B}, W) \subset F^{j} C(\mathcal{B}, W), \quad d F^{j} C(\mathcal{B}, W) \subset F^{j} C(\mathcal{B}, W) .
$$

Moreover, $\forall j \geq 1$ one has

$$
F^{q+j} C(\mathcal{B}, W) \cap C^{q}(\mathcal{B}, W)=0
$$

The boundedness property has many interesting consequences. For instance every cohomology space $H^{q}(\mathcal{B}, W)$ inherits a finite filtration by the images $H^{q}(\mathcal{B}, W)_{j}$ of $H^{q}\left(F^{j} C(\mathcal{B}, W) \cap C^{q}(\mathcal{B}, W)\right)$. 
According to our notations one has

$$
E_{0}^{q-j, j}=\frac{F^{j+1} C(\mathcal{B}, W) \cap C^{q}(\mathcal{B}, W)}{F^{j} C(\mathcal{A}, W) \cap C^{q}(\mathcal{B}, W)}=C^{q-j, j}(\mathcal{B}, W) .
$$

We summarize our discussion as follows:

Proposition 1. There is a spectral sequence $E_{r}^{p, q}$ such that

$$
E_{0}^{q-j, j}=C^{q-j, j}(\mathcal{B}, W), \quad E_{\infty}^{q-j, j}=\frac{H^{q}(\mathcal{B}, W)_{j}}{H^{q}(\mathcal{B}, W)_{j+1}} .
$$

The term $E_{1}^{\star, \star}$ is the relative cohomology space $\oplus_{q} H^{q}(\mathcal{B}, \mathcal{A}, W)$ which means that $H^{q}(\mathcal{B}, \mathcal{A}, W)=\oplus_{r+s=q} E_{1}^{r, s}$.

In the next subsection we point out some applications yielding some interesting spectral sequences which should lead to geometric invariants.

3.2. Bilagrangian structures ([HH], [NB1], [NB2], [WR1]). A bilagrangian structure on a symplectic manifold $(M, \omega)$ is a pair $\left(F_{1}, F_{2}\right)$ of lagrangian foliations which are transverse everywhere. Given a bilagrangian structure $\left(F_{1}, F_{2}\right)$ there is a unique torsion free symplectic connection $D$ which preserves both $F_{1}$ and $F_{2}$.

The pair $\left(F_{1}, F_{2}\right)$ is called locally (or affinely) flat if the curvature tensor of $D$ vanishes identically. In this case the $\mathrm{KV}$-algebras $\mathcal{A}_{i}=\mathcal{A}_{F_{i}}, i=1,2$ are left ideals of the $\mathrm{KV}$ algebra $\mathcal{A}$ of $(M, D)$. If $V$ is a two-sided $\mathcal{A}$-module then the cochain complexes $C^{\star}(\mathcal{A}, V)$, $C^{\star}\left(\mathcal{A}_{1}, V\right)$ and $C^{\star}\left(\mathcal{A}_{2}, V\right)$ are connected by spectral sequences. These considerations might yield new (bilagrangian) symplectic invariants. Indeed, every $\left(F_{1}, F_{2}\right)$-preserving symplectomorphism is a $(M, D)$-preserving diffeomorphism. We emphasize the role played by bilagrangian structures in the BKS method of geometric quantization [KB], [SJ], [SW]. This is another geometric reason why bilagrangian structures deserve to be deeply studied from the KV homology viewpoint.

4. Affinely flat Lie groups. A locally flat Lie group is a Lie group $\mathcal{G}$ endowed with a locally flat structure $(\mathcal{G}, D)$ which is invariant under the left translations by elements of $\mathcal{G}$. The vector space $\mathcal{X}(\mathcal{G})$ of smooth vector fields is a $\mathrm{KV}$-algebra whose multiplication is $X Y=D_{X} Y$. The subspace $g$ of left invariant vector fields on $\mathcal{G}$ is a subalgebra of the $\mathrm{KV}$-algebra $\mathcal{A}=(\mathcal{X}(\mathcal{G}), D)$. The following assertions are equivalent:

(a1) $J(g)=g$,

(a2) the linear connection $D$ is two-sided invariant.

Assertion (a2) means that the linear connection $D$ is invariant under the right translations as well. Actually $J(g)$ is an associative subalgebra of the $\mathrm{KV}$-algebra $g$, so we can equip the scalar complex $\oplus_{q} C^{q}(g, \mathbb{R})$ with the filtration

$$
F^{j} C(g, \mathbb{R})=\oplus_{q} F^{j} C(g, \mathbb{R}) \cap C^{q} C(g, \mathbb{R})
$$

where $\theta \in F^{j} C(g, \mathbb{R}) \cap C^{q}(g, \mathbb{R})$ iff $e\left(J(g)^{\otimes q-j+1}\right) \theta=0$. That is $\theta\left(b_{1}, . ., b_{q}\right)=0$ whenever the subset $b_{1}, . ., b_{q}$ contains more than $q-j+1$ arguments which belong to $J(g)$. The center $z(g)$ of the Lie algebra $g$ is contained in $J(g)$. Let aut $(g)$ be the space of infinitesimal 
automorphisms of the KV-algebra $g$. The first cohomology space $H^{1}(g, g)$ of the KValgebra $g$ is related to $J(g)$ by the exact sequence of vector spaces

$$
0 \rightarrow z(g) \rightarrow J(g) \rightarrow \operatorname{aut}(g) \rightarrow H^{1}(g, g) \rightarrow 0
$$

This exact sequence may be used to compute the dimension of the Lie group $A u t(\mathcal{G}, D)$ whose Lie algebra is $\operatorname{aut}(g)$. Really $\operatorname{Aut}(\mathcal{G}, D)$ is an isotopy subgroup of the group $\operatorname{Aff}(\mathcal{G}, D)$ of affine transformations of the locally flat manifold $(\mathcal{G}, G)$. Thus, the manifold $\mathcal{G}$ is the base of an $\operatorname{Aut}(\mathcal{G}, D)$-principal bundle whose total space is $\operatorname{Aff}(\mathcal{G}, A)$. Of course $\operatorname{Aut}(\mathcal{G}, D)$ is a closed subgroup of the Lie $\operatorname{Aut}(\mathcal{G})$ of automorphisms of the Lie group $\mathcal{G}$.

Here is a simple example. Consider the abelian Lie group $(\mathcal{G}, D)=\left(\mathbb{R}^{2}, D\right)$ where the two-sided invariant linear connection $D$ is defined by

$$
D_{(x, y)}\left(x^{\prime}, y^{\prime}\right)=\left(x x^{\prime}, 0\right) .
$$

One can easily check that $\operatorname{aut}(g)=H^{1}(g, g)=\mathbb{R} \theta$ with $\theta(x, y)=(o, y)$. Thus $\operatorname{dim} A f f(\mathcal{G}, D)$ $=3$

We consider another sample, $\mathcal{G}=\operatorname{af} f\left(\mathbb{R}^{2}\right)$. Its Lie algebra af $f\left(\mathbb{R}^{2}\right)$ has a canonical associative algebra structure. One has the following exact sequence

$$
0 \rightarrow \operatorname{aff}\left(\mathbb{R}^{2}\right) \rightarrow \operatorname{aut}\left(\operatorname{aff}\left(\mathbb{R}^{2}\right)\right) \rightarrow H^{1}\left(\operatorname{aff}(\mathbb{R}), \operatorname{aff}\left(\mathbb{R}^{2}\right)\right) \rightarrow 0 .
$$

We identify af $f\left(\mathbb{R}^{2}\right)$ with the subspace of $\mathcal{M}(3, \mathbb{R})$ consisting of the square matrices $\left[a_{i j}\right]$, $i, j=1,2,3$, such that $a_{3 j}=0, j=1,2,3$. Let $\omega$ be the exterior differential of the linear form $\theta$ defined by $\left[a_{i j}\right], i, j=1,2,3$,

$$
\theta\left(\left[a_{i j}\right]\right)=a_{11}+a_{12}+a_{22}+a_{32} .
$$

The form $\omega$ is a left invariant symplectic form on $A f f\left(\mathbb{R}^{2}\right)$. The Lie algebra af $f\left(\mathbb{R}^{2}\right)$ contains two lagrangian subalgebras which are transverse. They are defined by $\mathcal{A}_{1}: a_{11}=$ $a_{21}=a_{22}=0$ and $\mathcal{A}_{2}: a_{12}=a_{13}=a_{32}=0$ respectively. The pair $\left(\mathcal{A}_{1}, \mathcal{A}_{2}\right)$ defines a left invariant affinely flat bilagrangian structure on $\left(A f f\left(\mathbb{R}^{2}\right), \omega\right)$. The corresponding locally flat structure $\left(A f f\left(\mathbb{R}^{2}\right), D\right)$ differs from the canonical associative structure of aff $\left(\mathbb{R}^{2}\right)$. Indeed, on one hand both $\mathcal{A}_{1}$ and $\mathcal{A}_{2}$ are left ideals of the $\mathrm{KV}$-algebra $\left(\operatorname{aff}\left(\mathbb{R}^{2}\right), D\right)$. On the other hand $\mathcal{A}_{1}$ is not a left ideal of the associative subalgebra af $f\left(\mathbb{R}^{2}\right) \subset \mathcal{M}(3, \mathbb{R})$.

All the material we have just overviewed can be used to construct affine invariants. For instance let $(\mathcal{G}, D)$ be a locally flat Lie group. Let $d \mu$ be the left invariant Haar measure on $\mathcal{G}$. Let $W_{c}$ be the space of real valued compactly supported smooth functions on $\mathcal{G}$. It is a left module over the KV-algebra $g$. The canonical multiplication of real valued functions induces a pairing

$$
H^{q}\left(g, W_{c}\right) \times H_{q}\left(g, W_{c}\right) \rightarrow W_{c} .
$$

Then, to every pair $(\theta, \eta) \in H^{q}\left(g, W_{c}\right) \times H_{q}\left(g, W_{c}\right)$ is assigned a compactly supported function denoted by $\theta(\eta)$. We use the Haar measure to define a bilinear form

$$
B(\theta, \eta)=\int_{\mathcal{G}} \theta(\eta) d \mu
$$

This integral is invariant under the left translations on $\mathcal{G}$. 


\section{References}

[CL] F. Chapoton and M. Livernet, Pre-Lie algebra and the root tree operads, Internat. Math. Res. 8 (2001), 395-408.

[DA] A. Dzhumadldaev, Cohomologies and deformations of right symmetric algebras, J. Math. Sci. 93 (1999), 1836-1876.

[GM] M. Gerstenhaber, On deformation of rings and algebras, Ann. of Math. 79 (1964), 59-103.

[GLS] V. Guillemin, E. Larman and S. Sternberg, Symplectic Fibrations and Multiplicity Diagram, Cambridge University Press, 1996.

[HH] H. Hess, Connections on symplectic manifolds and geometric quantization, in: Lect. Notes in Math. 836, Springer, Berlin, 153-165.

[HS] G. Hochschild and J.-P. Serre, Cohomology and Lie algebras, Ann. of Math. 3 (1953), 591-603.

[KH] H. Kim, Complete left invariant affine structure on nilpotent Lie groups, J. Diff. Geom. 24 (1986), 373-394.

[KB] B. Kostant, Quantization and unitary represenatations. Prequantization Part I, in: Lect. Notes in Math. 170, Springer, 1970, 87-208.

[KJL] J.-L. Koszul, Déformations des connexions localement plates, Ann. Inst. Fourier 18 (1968), 103-114.

[MA] S. A. Merhulov, Nijenhuis infinity and contractible graded differential manifolds, Comp. Math. 141 (2005), 1238-1254.

[MJ] J. Milnor, On the fundamental group of affinely flat manifolds, Adv. in Math. 47 (1975), 223-228.

[NB] M. Nguiffo Boyom, Algèbres à associateur symétrique et algèbres de Lie reductives, Thèse Spec., Fac. Sci. Grenoble, 1968.

[NB1] M. Nguiffo Boyom, Métriques Kähleriennes affinement plates, Proc. London Math. Soc. 3 (1993), 358-380.

[NB2] M. Nguiffo Boyom, Structures localement plates dans certaines variétés symplectiques, Math. Scand. 76 (1995), 61-84.

[NB3] M. Nguiffo Boyom, The cohomology of Koszul-Vinberg algebras, Pacific J. Math. 225 (2006), 119-153.

[NB4] M. Nguiffo Boyom, Cohomology of Koszul-Vinberg algebroids and Poisson manifolds I, in: J. Kubarski et al. (eds.), Lie Algebroids and Related Topics in Differential Geometry, Banach Center Publ. 54, 2001, 99-110.

[NB5] M. Nguiffo Boyom, Cohomology of Koszul-Vinberg algebroids and Poisson manifolds II, Inter. J. of Math. 16 (2005), 1-29.

[NB6] M. Nguiffo Boyom, KV-Homology of lagrangian foliations, in preparation.

[NBW1] M. Nguiffo Boyom and R. Wolak, Affine structure and KV-cohomology, J. Geom. Phys. 42 (2002), 307-317.

[NBW2] M. Nguiffo Boyom and R. Wolak, Local structure of Koszul-Vinberg and of Lie algebroids, Bull. Sci. Math. 128 (2004), 467-479.

[NA] A. Nijenhuis, Sur une classe de propriétés communes à quelques types différents d'algèbres, Ens. Math. 14 (1969), 225-277.

[SW] D. Simms and N. Woodhouse, Geometric Quantization, Lect. Notes in Physics 53, Springer, 1976.

[SJ] J-M. Souriau, Structure des systèmes dynamiques, Dunod, Paris, 1970. 
[WR1] R. Wolak, Ehresmann connections for lagrangian foliations, J. Geom. Phys. 17 (1995), 310-320.

[WR2] R. Wolak, Transversely affine foliations compared with affine manifolds, Quart. J. Math. Oxford 41 (1990), 369-384. 
